\title{
Cancer communications for the development of personalized medicine
}

\author{
Kaiser Jamil \\ Genetics Department, Bhagwan Mahavir Medical Research Center, Hyderabad-500004, A.P., India \\ Correspondence: Kaiser Jamil. Address: Genetics Department, Bhagwan Mahavir Medical Research Center, \\ Hyderabad-500004, A.P., India. Telephone: 91-967-687-2626. Fax: 91-406-663-1500. E-mail: kj.bmmrc@gmail.com \\ Received: January 10, 2012 \\ Accepted: March 15, 2012 \\ Published: April 1, 2012 \\ DOI : $10.5430 /$ jst.v2n2p1 \\ URL: http://dx.doi.org/10.5430/jst.v2n2p1
}

\section{Introduction}

Keeping in view the need for corporate communications in cancer research perspective, which means communications serves as the liaison between an organization and public. This editorial presents a view which is very different from commercial-marketing a product or laisoning with producer companies etc. It is all about developments in cancer research which leads one to understand - what is personalized medicine. As the scientific and medical communities begin to decipher the secrets locked in the human genome code, the way we approach the diagnosis, treatment and prevention of disease will change dramatically. With the detection of human genome, scientists have already created large databases filled with thousands of single nucleotide sequence changes. Some of these single nucleotide polymorphisms (SNPs) could define the genetic basis of what keeps healthy or makes us sick. Currently the most common applications for SNP related research tools are gene-disease association studies, drug-gene interactions and drug target validation. Other popular applications are disease susceptibility studies or diagnostics, pharmacogenomic studies for clinical trials, drug target screening, and new technology development.

The revolution in cancer research can be summed up in a single sentence: Cancer is, in essence, a genetic disease involving more than 350 signature genes distributed over several chromosomes. There is now evidence that alterations in three types of genes are responsible for tumorigenesis: oncogenes, tumor-suppressor genes and stability genes. Unlike diseases like sickle cell anemia, cystic fibrosis or muscular dystrophy, wherein mutations in one gene can cause the disease, no single gene defect 'causes' cancer. Human cells have multiple safeguards to protect them against the potentially lethal effects of cancer gene mutations, and only when several genes become defective then an invasive cancer develops, this phenomenon explains the functional network of cancer metasignature genes. Thus it is best to think of mutated genes as contributing, rather than causing, cancer.

\section{Research, genetic differences affecting patient's treatment response}

Our work involves an ongoing quest to identify genomic factors that control drug response in individuals and populations; the bibliographic list below shows our contributions in this area for the last seven years. Clinical observations of inherited differences in drug effects were first documented in the 1950s, giving rise to the field of Pharmacogenomics. The figure below is an excellent example of distribution of phenotypes with respect to the various effects of one drug given to a group of patients diagnosed with similar clinical symptoms. 


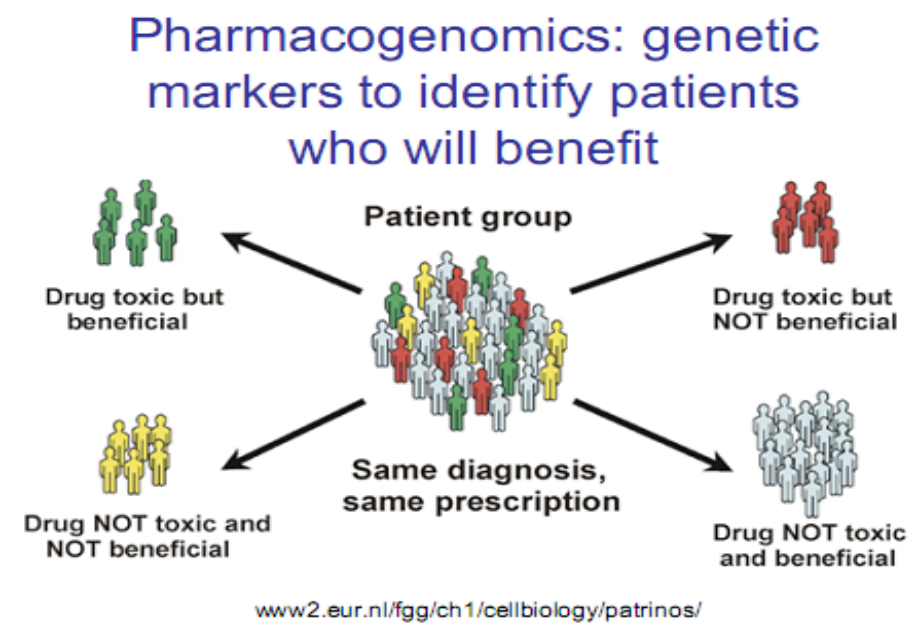

Figure 1. Distribution of phenotypes with respect to the various effects of one drug given to a group of patients diagnosed with similar clinical symptoms

Pharmacogenomics is a rapidly growing field that aims to elucidate the genetic basis for inter-individual differences in drug response and to use such genetic information to predict the safety, toxicity and/or efficacy of drugs in individual patients or groups of patients. While drug-gene interactions and environmental factors significantly contribute to inter-individual variability in drug response, genetic factors also appear to have a major impact on drug response and disposition. Considering the significant heterogeneity associated with patient responses to chemotherapeutic agents and their narrow therapeutic indices, pharmacogenomics has the potential to offer individualized cancer treatment regimens. Although many non-genetic factors influence the effects of medications, including age, environmental factors, habits, organ function, concomitant therapy, drug interactions (as shown in the diagram below) and the nature of the disease, there are now numerous examples of cases in which inter-individual differences in drug response are due to sequence variants in genes encoding drug-metabolizing enzymes, drug transporters, or drug targets. Such studies can be translated to clinical practice via molecular diagnostics (genotyping) in order to guide selection of the optimal drug combination and dosage for the individual patient.

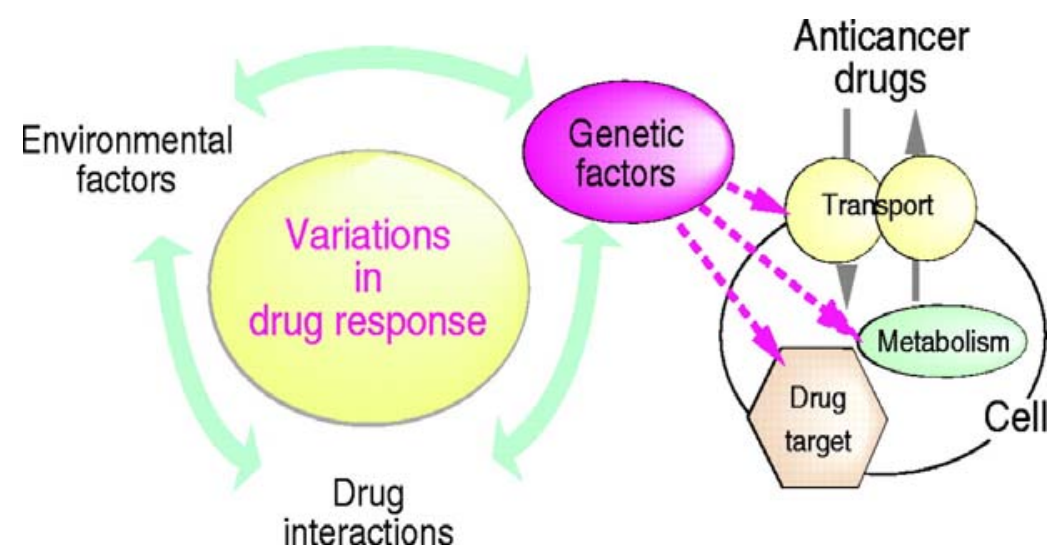

Figure 2. Multiple factors contributing to variations in drug responses

\section{Theory and practice (Pharma market trends)}

The recent trends in Pharmacogenomics will begin to increase steadily and could quickly become a multibillion-dollar market itself. Every year as many as 100,000 patients in hospitals die as the result of adverse drug reactions. How many of those deaths could be avoided if we knew in advance which drugs were safe and which weren't for those particular 
patients? And beyond those dire cases, how much more effectively could we treat sick people if we knew what variants in their genomes made certain drugs more effective than others for them? The rapidly developing field of Pharmacogenomics aims to examine the genomes (DNA) of patients and use that information to prescribe the right drugs at the right dose. The knowledge of the expression of genes is critical to the action of the cancer drugs that can be used to individualize therapy.

In every cancer diagnosis, communication plays a key role in helping patients and physicians make the best decisions about treatment options, risks, and benefits. And those who live beyond cancer are more likely to experience a better quality of life when they have access to useful information and support in dealing with post-treatment effects. Culturally and linguistically appropriate communication helps people make decisions that are compatible with people's values and beliefs. The goal of communications in cancer research is to first Understand, Apply, and Disseminate effective communication approaches that maximize access for appropriate use of cancer information by all those who need it.

\section{References}

[1] Suman G and Kaiser Jamil, (2006) Novel CYP3A4 gene polymorphisms in post chemo breast cancer patients, International Journal of Cancer Research 2,(4)358-66. http://dx.doi.org/10.3923/ijcr.2006.358.366

[2] Suman G and Kaiser Jamil (2006) Studies on Combination Vs. Single Anti-Cancer Drugs In vitro, Perspectives in Cytology and Genetics, 12;185-95.

[3] Kalyan Kumar and Kaiser Jamil (2006) "Methylenetetrahydrofolatereductase (MTHFR) C677T and A1298C polymorphisms and breast cancer in South Indian population" International Journal of Cancer Research. 2(2)143-51. http://dx.doi.org/10.3923/ijcr.2006.143.151

[4] Haranatha Reddy, \& Kaiser Jamil, (2006) Polymorphisms in the MTHFR gene and their possible association with susceptibility to childhood acute lymphocytic leukemia (ALL) in Indian population: Leukemia and Lymphoma, 47(7)1333-39. http://dx.doi.org/10.1080/10428190600562773

[5] Haranatha Reddy \& Kaiser Jamil. (2006) Polymorphism in the GST (M1 and T1) gene and their possible association with susceptibility to childhood acute lymphocytic leukemia (ALL) in the Indian population. African J. of Biotechnology, 5,(16)1454-56.

[6] Haranatha Reddy, Sudhamurthy and Kaiser Jamil (2006) “Association of FLT3-ITD (Internal Tandem duplication) polymorphism in the childhood acute lymphocytic leukemia, Recent advances in biotechnology, Sep 2006, pp192-98.

[7] Rama Mani S, Sudha Murthy and Kaiser Jamil.(2006) Role of serum Lactate dehydrogenase as a bio-Marker in Therapy related Hematological malignancies International Journal of Cancer Research, 2(4)383-89. http://dx.doi.org/10.3923/ijcr.2006.383.389

[8] Kaiser Jamil and Haranatha Reddy (2007): Can Polymorphisms in genes relate to overall survival in leukemias? Leukemia and Lymphoma 2007,48,6;1070-71. http://dx.doi.org/10.1080/10428190701287353

[9] Rama Mani, Kaiser Jamil and M. ChVamsy (2007): Specificity of serum tumor Markers (CA125, CEA, AFP, Beta HCG) in Ovarian Malignancies. Trends in Medical Research, 2(3),128-34. http://dx.doi.org/10.3923/tmr.2007.128.134

[10] Siva Shanker, Satish Chandra Reddy, Kaiser Jamil \& M.Ch. Vamsy (2007). Genomic analysis of SNPs in Breast Cancer by using Bioinformatics databases. Ind. J. Biotech, Vol.6,pp456 -62.

[11] Kalyana Kumar Ch, Sudha Murthy and Kaiser Jamil (2007) "Possible Association of Splice Site Mutation of Dihydropyrimidine Dehydrogenase (IVS14+1G>A) in Adverse Drug Reactions in Some Invasive Ductal carcinoma Patients. International J. of Pharmacology.2007,3(2)130-36.

[12] Shaswati Khan, Kaiser Jamil, Prabhavathy Das, Mohana Vamsy Ch and Sudha M. (2007) Polymorphic sites (1236 and 3435$)$ in mdr1 gene influencing drug response in breast cancer patients. International Journal of Pharmacology 3(6):453-60. http://dx.doi.org/10.3923/ijp.2007.453.460

[13] K. Sabitha, M. V.V. Reddy and Kaiser Jamil; (2008) GST genotypes in head and neck cancer patients and its clinical implications. African Journal of Biotechnology. November, 2008,Vol.7(21),pp5. Available online at http://www.academicjournals.org/AJB.

[14] Kaiser Jamil, Kalyan Kumar, S. Hajira Fatima, Syed Rabbani, Ravi Kumar, Ramesh Perimi (2009). Clinical Studies on Hormonal Status in Breast Cancer and its Impact on Quality of Life (QOL). Journal of Cancer Science and Therapy (JCST) /Vol.1.283-89.

[15] Sabitha K, Vishnu Vardhan Reddy M, and Kaiser Jamil (2009) Mutations in XRCC1 gene altering the genetic risks of head and neck cancer patients. International Journal of cancer research.2009,5(2),58-68. http://dx.doi.org/10.3923/ijcr.2009.58.68

[16] Shaswati Khan and Kaiser Jamil 2009, Linkage disequilibrium analysis determines the association of the haplotypes of MDR1 with IDC breast cancer, International Journal of Genetics and Molecular Biology Vol.1(6),pp105-14.

[17] Kalyana Kumar, MohanaVamsy and Kaiser Jamil, 2010, Thymidylate synthase gene polymorphisms effecting 5-FU response in breast cancer patients. Cancer Biomarkers 5/2010,1-11.

[18] Sabitha, M. Vishnuvardhan Reddy, Kaiser Jamil, 2010, Smoking related risk involved in individuals carrying genetic variants of CYP1A1 gene in head and neck cancer. Cancer Epidemiology - 2010 Oct; 34(5):587-92 (International Journal of Cancer Epidemiology, Detection, and Prevention. CANEP-). PMid:20887941. http://dx.doi.org/10.1016/j.canep.2010.05.002

[19] Balaji A.B., Kaiser Jamil, Maruti Ram and Habibulla C.M. -2010-Isolation of a novel population of multipotent stem cells from epidermal layer of human skin; Biology and Medicine, Vol.2(2):57-67.

[20] M Ahmed and K Jamil, 2011, Cytotoxicity of neoplastic drugs Gefitinib, Cisplatin, 5-FU, Gemcitabine, and Vinorelbine on human cervical cancer cells (HeLa). Biology and Medicine, 3(5):60-71. 